\title{
ACTITUDES HACIA LA SEXUALIDAD DE LAS PERSONAS CON DISCAPACIDAD INTELECTUAL: ¿DIFIERE LA POBLACIÓN GENERAL DE LAS PROPIAS PERSONAS CON DISCAPACIDAD INTELECTUAL?
}

\author{
Nicola-Piris, Y. \\ Dpto. de Psicología Evolutiva y de la Educación \\ Universidad de Valencia, Valencia, España \\ Yarisa.nicola@uv.es \\ Gil-Llario, M. D. \\ Dpto. de Psicología Evolutiva y de la Educación \\ Universidad de Valencia, Valencia, España \\ Morell-Mengual, $\mathbf{V}$. \\ Dpto. de Psicología Evolutiva y de la Educación \\ Universidad de Valencia, Valencia, España \\ Fernández-García, 0. \\ Dpto. de Psicología Evolutiva y de la Educación \\ Universidad de Valencia, Valencia, España
}

\author{
Recepción Artículo: 13 marzo 2020 \\ Admisión Evaluación: 17 marzo 2020 \\ Informe Evaluador 1: 1 abril 2020 \\ Informe Evaluador 2: 7 abril enero 2020 \\ Aprobación Publicación: 20 abril 2020
}

Esta investigación ha sido realizada en el marco del proyecto de investigación "Desarrollo y análisis de la eficacia de un programa de educación afectivo-sexual para prevenir el abuso y mejorar la salud sexual en personas con diversidad funcional intelectual" financiado por el Programa Estatal de I+D+i Orientada a los Retos de la Sociedad del Ministerio de Ciencia e Innovación (Proyecto RTI2018-095538-B-100).

\section{RESUMEN}

Un aspecto esencial para garantizar la calidad de vida en las personas con Discapacidad Intelectual (PDI) es la salud sexual. Pese a que en los últimos años se ha aumentado la atención en esta cuestión, es determinante conocer si existen prejuicios que pueden afectar al desarrollo saludable. El objetivo de este estudio ha sido comparar las actitudes hacia la sexualidad de las PDI que tienen ellas mismas con las que tiene la población general (PPG). Para ello un total de 100 PDI (50\% hombres y 50\% mujeres), de 19 a 55 años completaron la Escala de Percepción Sexual, y 454 PPG (39.4\% hombres y 60.6\% mujeres), de entre 20-82 años completaron la Escala de Actitudes para la Sexualidad en Personas con Discapacidad Intelectual (ASEXDI). El 71.1\% de las PPG presenta unas actitudes moderadas hacia la sexualidad de las PDI, el $18.7 \%$, actitudes liberales y $10.1 \%$ conservadoras. Ambos grupos difieren en la tolerancia a las caricias siendo superior en el grupo de PPG (68.28\% lo con- 
sidera muy aceptable, frente al $22.78 \%$ de las PDI). Por el contrario, las relaciones sexuales con penetración entre PDI están ligeramente mejor valoradas por las PDI que entre la PG, aunque en ambos casos son actitudes conservadoras (el 15\% de las PDI lo considera nada aceptable en absoluto, frente al $37.89 \%$ de la PG). Sin embargo, la PG tiende a considerar de forma más positiva las competencias de las PDI para el uso correcto del preservativo. Los resultados permiten concluir que la PG muestra una tolerancia moderada hacia la expresión de la sexualidad de este colectivo, con la excepción de la práctica de penetración hacia la que muestran cierta prevención. La actitud positiva de Ios PDI hacia esta práctica unida a su baja percepción de competencia en el uso del preservativo justifica la necesidad de programas de educación afectivo-sexual adaptados.

Palabras clave: actitudes; sexualidad; diversidad funcional intelectual; discapacidad; parentalidad en diversidad intelectual

\begin{abstract}
Attitudes towards sexuality of people with intellectual disabilities: Does the general population differ from own persons with intellectual disabilities? An essential aspect to guarantee the quality of life in people with Intellectual Disabilities (PDI) is sexual health. Despite the fact that attention has been increased in recent years on this issue, it is decisive to know if there are prejudices that can affect healthy development. The objective of this study has been to compare the attitudes towards sexuality of PDI that they have with those of the general population (PPG). For this, a total of $100 \mathrm{PDI}$ (50\% men and 50\% women), from 19 to 55 years old, completed the Sexual Perception Scale, and 454 PPG (39.4\% men and 60.6\% women), between 2082 years old, completed the Attitude Scale for Sexuality in People with Intellectual Disabilities (ASEXDI). 71.1\% of the PPG show moderate attitudes towards the sexuality of PDI, 18.7\%, liberal and $10.1 \%$ conservative attitudes. Both groups differ in tolerance to caresses, being higher in the PPG group (68.28\% consider it very acceptable, compared to $22.78 \%$ of $\mathrm{PDI}$ ). In contrast, penetrative sexual relations between PDI are slightly better valued by PDI than between PPG, although in both cases they are conservative attitudes (15\% of PDI consider it not at all acceptable, compared to $37.89 \%$ of the PPG). However, the PPG tends to consider the competences of PDI for the correct use of condoms in a more positive way. The results allow to conclude that the PPG shows a moderate tolerance towards the expression of sexuality of this group, with the exception of the penetration practice towards which they show some prevention. The positive attitude of PDI towards this practice together with their low perception of competence in condom use justifies the need for adapted affective-sexual education programs.
\end{abstract}

Keywords: attitudes; sexuality; intellectual functional diversity; disability; parenting in intellectual diversity

\title{
INTRODUCCIÓN
}

La discapacidad intelectual (DI) constituye un trastorno del neurodesarrollo caracterizado por deficiencias en las funciones intelectuales, en resolución de problemas, pensamiento abstracto, planificación, juicio, razonamiento, aprendizaje a partir de la experiencia y aprendizaje académico; y deficiencias en el comportamiento adaptativo, que impiden la consecución de los estándares sociales y culturales para la independencia personal y la responsabilidad social y limitan el funcionamiento en una o más actividades de la vida diaria, tales como la participación social, la comunicación y la vida independiente (APA, 2013).

La situación social de las PDI ha sido una historia de segregación a lo largo de los años hasta el siglo XIX, pero la integración en la sociedad ha sido lenta y desigual (Forrai, 2020), con una mayor apertura hacia aspectos culturales y laborales (Álvarez, Vega, Spencer, González y Arriagada, 2019) y mucho menor hacia aquellos aspectos relacionados con la vida sexual y las emociones de las PDI (Morell-Mengual, Gil-Llario, Díaz-Rodríguez y Caballero-Gascón, 2017).

El entorno en el que crecen las PDI influye, de forma determinante, en su desarrollo y en cómo se desenvuelven (Mena-Chávez y Martínez-Herrera, 2019). El Modelo Social de la Diversidad Funcional nació hacia finales de los años 60 del siglo pasado en Estados Unidos (Wehmeyer 1999, 2005) y pretendió cambiar el foco de la individualidad tradicional que caracterizaba a las PDI a un enfoque más social donde se remediaran las caren- 
cias con inserción e interacción social (Velarde-Lizama, 2012). Así, en las últimas décadas, ha habido un aumento significativo de la presencia activa de las PDI en la sociedad, gracias a este enfoque más centrado en la capacidad de los jóvenes con PDI para asistir a la educación general y vivir su vida plenamente (Banks, McCoy, Frawley, Kingston, Sheylin y Smyth, 2016). De igual modo, estos cambios hacia un modelo de atención biopsicosocial y de igualdad en el acceso a la educación, han hecho que, cada vez más, se considere esencial una educación centrada en las relaciones íntimas y la sexualidad dirigida a este colectivo (Forrai, 2020).

La sexualidad es un aspecto central del ser humano presente a lo largo del ciclo vital, que abarca el sexo, los roles de género, la identidad, el placer y el erotismo (Morell-Mengual, Gil-Llario, Díaz-Rodríguez y CaballeroGascón, 2017). En la actualidad diversos estudios revelan que la sexualidad está profundamente influenciada por las actitudes. Cabe destacar que las actitudes, las creencias y los valores hacia la sexualidad se elaboran y mantienen en el marco de la familia, los amigos, los grupos de referencia, la sociedad y la cultura, e influyen en la realización de comportamientos sexuales de riesgo (Díaz-Rodríguez, Gil-Llario, Morell-Mengual, SalmerónSánchez y Ruíz-Palomino, 2016).

Se observa que gran parte de este colectivo presenta deseos de poder hablar de forma más abierta sobre temas relacionados con el vínculo afectivo y la parentalidad (Gil-Llario, Morell-Mengual, Ballester-Arnal y DíazRodríguez, 2018) ya que, como se ha constatado, presentan las mismas necesidades a nivel afectivo-sexual que las personas sin discapacidad (Egholm, 2015; Borawska-Charko, Rohleder y Finlay, 2017). No obstante, han sido tradicionalmente consideradas como personas no aptas para la expresión libre de su sexualidad, mantener relaciones afectivo-sexuales o vivir en pareja (Díaz-Rodríguez, Gil-Llario, Ballester-Arnal, Morell-Mengual, MoleroMañes, 2014). La sexualidad de estas personas se ha visto influenciada por una serie de prejuicios, mitos y falsas creencias en la población general (Isler, Tas, Beytut y Conk, 2009; Pownall, Jahoda y Hastings, 2012; Coyne, 2016). Y, pese a estar descrito como un derecho fundamental en la Convención Internacional de los Derechos de las Personas con Discapacidad (Olavarrieta, Darín, Suárez, Besteiro y Gómez-Jarabo, 2013), la investigación en este sentido sigue siendo reducida (Deffew, 2019). Esto nos lleva a pensar que existe una distancia entre el reconocimiento de este derecho y el ejercicio efectivo del mismo (Rojas-Pernia, Haya-Salmón y Lázaro-Visa, 2016).

La información que se les proporciona es sustancialmente menor, y los apoyos que se facilitan para que alcancen una vida normalizada a nivel afectivo-sexual son insuficientes; es como si esa información que se les proporciona se quedara "congelada" en el tiempo y siempre fueran niños (Forrai, 2020). Por tanto, pese a los movimientos sociales y campañas de concienciación de asociaciones y organismos, existe una tendencia a asexualizar a este colectivo y calificarlos como incapaces de participar en actividades sexuales, reduciéndoles, por ejemplo, el acceso a información o negándoles la parentalidad (Powell y Stein, 2016). Esto se muestra contrario a lo que promueve la Organización Mundial de la Salud (2015) que considera que la capacidad de formar relaciones personales y sexuales, también en este colectivo, está fuertemente relacionada con el bienestar y felicidad.

Estudios recientes señalan que los profesionales y padres están cada vez más abiertos a la expresión de la identidad sexual de las PDI (Deffew, 2019). Sin embargo, la investigación continúa reconociendo que aún tienen sus limitaciones ( wirynkalo, Byra y yta, 2017; Brown y McCann, 2018). En esta línea, se observa que suelen presentar actitudes moderadas-liberales hacia diferentes aspectos de la sexualidad de las PDI, pero que estas actitudes son menos positivas que las que muestran hacia la sexualidad de las personas sin discapacidad (Sankhla y Theodore, 2015).

Así, parece ser que la sociedad aún mantiene una postura muy protectora hacia la expresión de la sexualidad de las PDI (Rushbrooke, Murray y Townsend, 2014), por tanto, el objetivo de esta investigación será comparar las actitudes hacia la sexualidad de las PDI que tienen ellas mismas con las que tiene la PG. 


\section{ACTITUDES HACIA LA SEXUALIDAD DE LAS PERSONAS CON DISCAPACIDAD INTELECTUAL: ¿DIFIERE LA POBLACIÓN GENERAL DE LAS PROPIAS PERSONAS CON DISCAPACIDAD INTELECTUAL?}

\section{MÉTODO}

\section{Participantes}

La muestra está constituida por un total de 814 personas, divididas en dos grupos. El primer grupo está conformado por 360 sujetos con discapacidad intelectual (DI) y edades comprendidas entre 19 y 55 años (50\% mujeres y $50 \%$ hombres). Por lo que respecta a la edad, un $14.2 \%$ tiene entre $19-25$ años, un $15.5 \%$ entre $26-35$, un $36.1 \%$ entre $36-45$ años y un 34.2\% entre 46-55 años. Y en lo referido al Cociente Intelectual (Cl), se observa que el $58.7 \%$ de las PDI está entre un 50-70 y el resto, un 41.1\% tiene un $\mathrm{Cl}$ entre $71-85$.

El segundo grupo de población general está formado por 454 personas de entre 20 y 82 años (39.4\% hombres y $60.6 \%$ mujeres). El mayor grupo lo conforman personas de entre $20-35$ años (63.4\%), el $21.4 \%$ se halla entre los 36-51, el 14.3\% entre los 52-66 años y el 0.9\% entre los 67-82 años. En cuanto al nivel de formación, el $2.6 \%$ no posee estudios, el $18.7 \%$ graduado escolar, el $24.7 \%$ educación secundaria y el $54 \%$ educación superior.

Los únicos criterios de inclusión para el reclutamiento de la muestra de las PPG fue que residieran en España, fueran mayores de 18 años y tuvieran un dominio de la lectura del español suficiente para comprender adecuadamente las preguntas. En cuanto a la población con DI, Ios criterios de inclusión consistían en poseer un certificado de discapacidad intelectual leve o moderada, contar con suficientes competencias verbales para poder responder adecuadamente al cuestionario que se les solicitaba y tener capacidad para dar el consentimiento para participar de forma libre.

\section{Instrumentos}

ASEXDI. Escala de Actitudes hacia la Sexualidad en Personas con Discapacidad Intelectual (Gil-Llario, Fernández-García, Castro-Calvo, Caballero-Gascón y Ballester-Arnal, en revisión). Cuestionado compuesto de 18 ítems, cuyo objetivo es evaluar las actitudes que se poseen en relación a la sexualidad de las PDI. Las respuestas son presentadas en una escala tipo Likert con cinco opciones de respuesta que van desde 1 (totalmente de desacuerdo) hasta 5 (totalmente de acuerdo). Concretamente en esta investigación se han seleccionado tres ítems relacionados con la conducta sexual: besos y caricias «me parece bien que las PDI se besen 0 acaricien con otra persona», sexo sin penetración «me parece bien que las PDI tengan relaciones sexuales siempre que no haya penetración» y coito vaginal «me parece bien que las PDI tengan relaciones sexuales con o sin penetración». Esta escala permite obtener una puntuación global sumando las respuestas de cada ítem. La puntuación mínima será de 3 puntos y la máxima de 15, por lo que una puntuación más elevada, sugiere actitudes más erotofílicas respecto a la sexualidad. Así, para interpretar los datos, se han establecido tres rangos: actitudes conservadoras o erotofóbicas (de 3-6 puntos); actitudes moderadas (de 7-11 puntos); y actitudes liberales 0 erotofílicas (de 12-15 puntos). Para obtener la puntuación global, se calcula realizando un sumatorio de los puntos asignados por cada ítem, el cual oscila entre 18 y 90 . En el estudio realizado por DíazRodríguez (2017), se obtuvo un coeficiente de fiabilidad alfa de Cronbach de .71 para la población general.

POS. Escala de percepción sexual para personas con discapacidad intelectual (Scotti, Slack, Bowman y Morris, 1996). Escala formada por un total de 28 ítems que evalúa comprensión, conocimientos e ideas, positivas y negativas, sobre diferentes comportamientos y prácticas sexuales para parejas del mismo sexo y de diferente sexo. El formato de respuesta es tipo Likert 1-5 (nada-aceptable). Concretamente para la realización de este estudio, se han seleccionado 3 ítems relacionados con tres prácticas sexuales: besos «beso prolongado con un compañero de diferente sexo", sexo oral «sexo oral con un compañero de diferente sexo»y coito vaginal «coito vaginal con un compañero de diferente sexo». La escala ofrece la posibilidad de realizar puntuaciones por cada ítem, así como una puntuación global de 3 a 15, al sumar el valor de las respuestas. 


\section{Procedimiento}

Tras obtener la aprobación del Comité de Ética de la Universitat de Valencia, se contactó con los responsables de los servicios de apoyo para las PDI de las comunidades autónomas españolas de Andalucía, Comunidad Valenciana y Castilla-La Mancha para solicitarles su participación en el estudio. Se contactó vía telefónica con los centros participantes para ofrecer información sobre los objetivos del estudio, los criterios de inclusión y, una vez aceptada la participación, para concretar el procedimiento de recogida de datos.

El reclutamiento de la muestra de las PPG se realizó mediante el método bola de nieve, solicitando a padres y profesionales que trabajan con usuarios con DI que lo difundieran entre sus conocidos, sugiriéndoles también que lo difundieran, a su vez, entre sus conocidos.

\section{Análisis de datos}

Con el fin de analizar los datos aportados por la muestra, se realizaron análisis estadísticos descriptivos, pruebas $t$ de Student y ANOVAS, mediante el programa IBM SPSS Statistics 23.

\section{RESULTADOS}

El 71.1\% de las PPG presentan actitudes moderadas, el 18.7\% actitudes más liberales y un 10.1\% actitudes más conservadoras hacia la sexualidad de las PDI. En cuanto a las actitudes de las PDI, el grupo más numeroso es el que presenta actitudes más moderadas (55.3\%), seguido de los que presentan actitudes conservadoras (36.4\%) y liberales (8.3\%) (Ver Figura 1).

Figura 1 Actitudes hacia la sexualidad de las personas con discapacidad

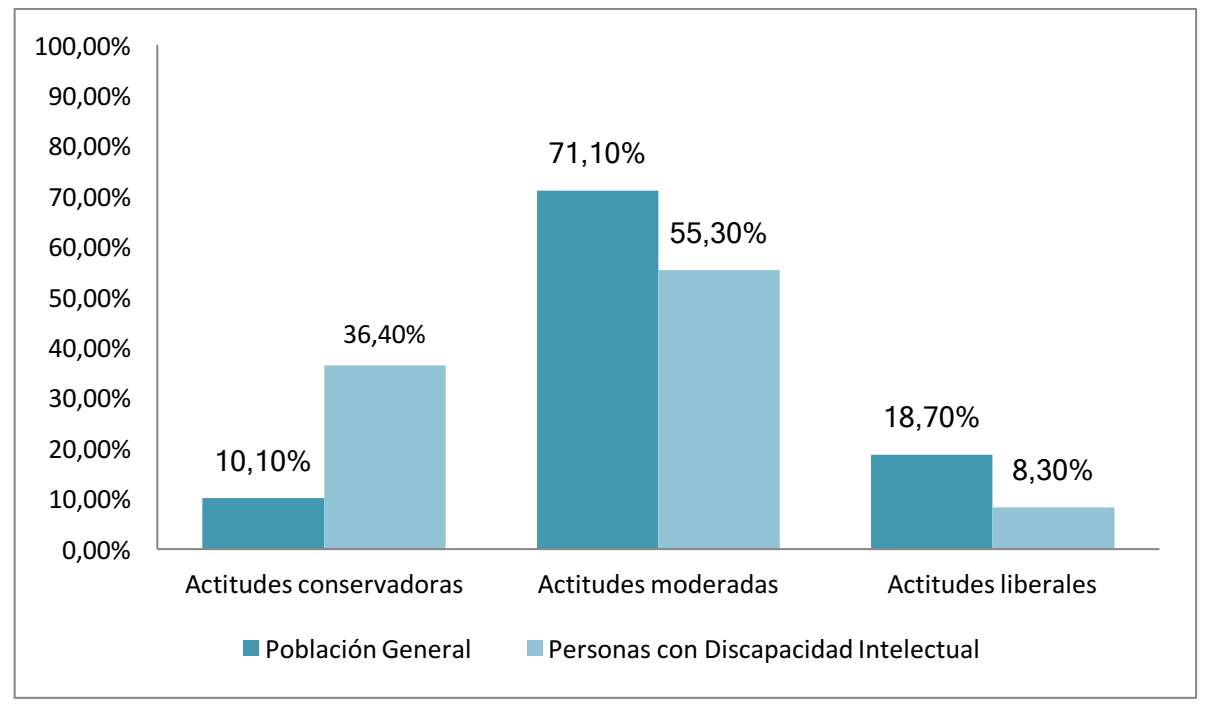

Al analizar el sumatorio de los 3 ítems, se observa que la PG presenta una puntuación media superior ( $M=$ 10.05; $\mathrm{DT}=2.31)$ respecto a las PDI $(M=8.35 ; \mathrm{DT}=2.16)$, mostrando diferencias estadísticamente significativas entre las puntuaciones globales de ambos grupos $(t=-10.70 ; p=.000)$.

En cuanto a la tolerancia a besos y caricias entre PDI, la PG se muestra más favorable ya que más de la mitad de los encuestados Io califica como "muy aceptable" (68.28\%), mientras que las PDI presentan unas actitudes más conservadoras pues el $32.22 \%$ considera que son conductas sexuales "nada aceptables" ( $X^{2}=243.45$; $\mathrm{p}=.000$ ) (Ver figura 2). 
Figura 2 Actitudes hacia los besos y caricias entre las personas con DI.

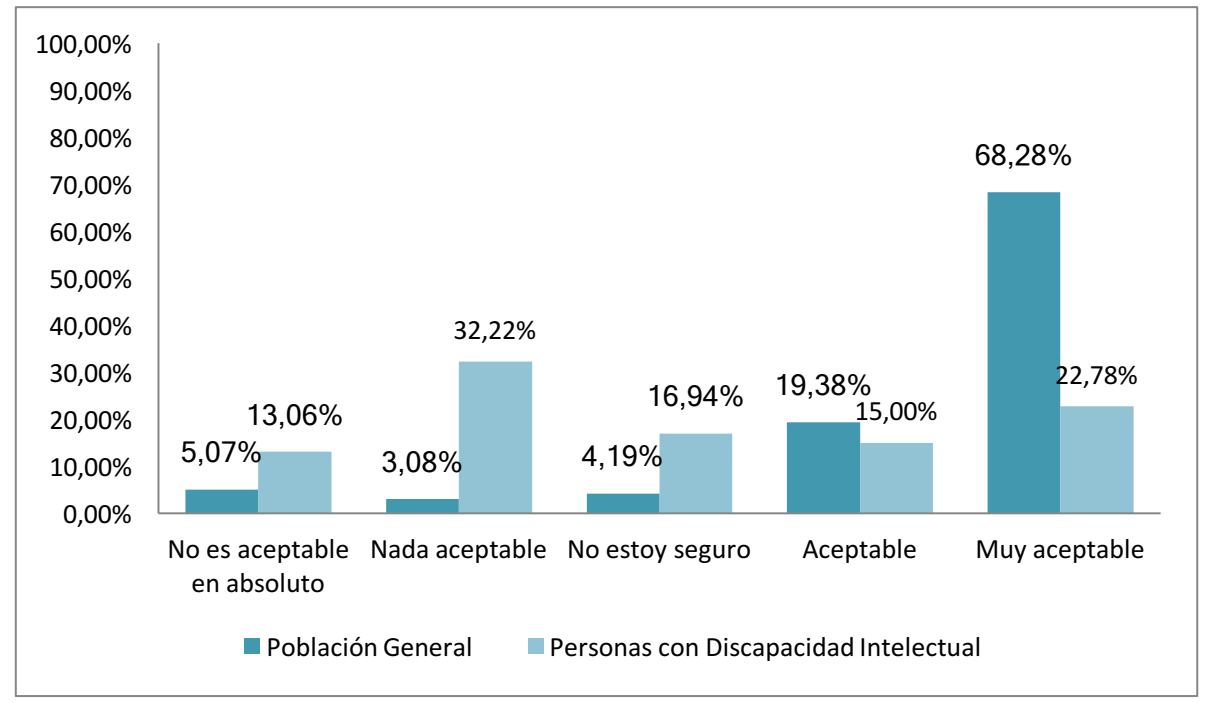

En cuanto a la tolerancia a las relaciones sexuales sin penetración entre PDI, se muestra una tendencia mucho más conservadora en ambos grupos que en el ítem anterior. Contrariamente, el 37.89\% de la PG lo califica como "no aceptable en absoluto", frente al 15\% de las PDI. Esto indica que, en este punto, las PDI no son tan conservadoras, pero, aun así, encontramos que la mayoría (39.72\%) califica estas conductas sexuales como "no aceptable" ( $\left.X^{2}=74.35 ; p=.000\right)$ (Ver figura 3$)$.

Figura 3 Actitudes hacia relaciones sexuales sin penetración entre personas con DI.

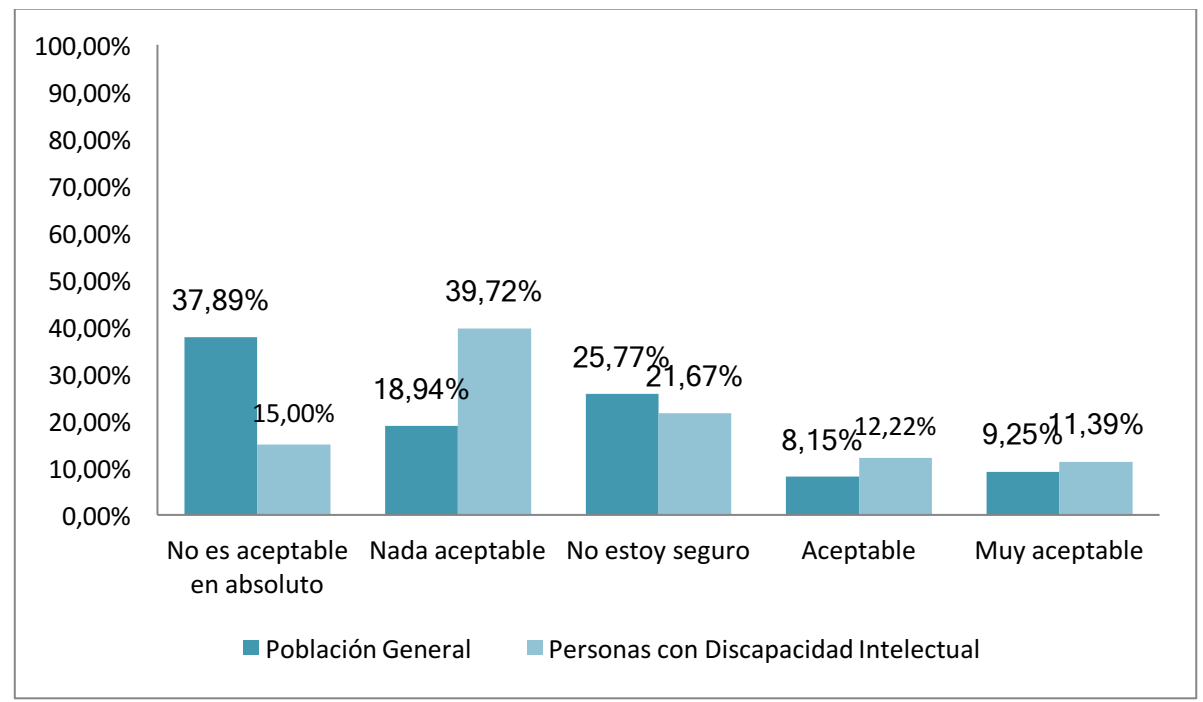


En cuanto a la percepción del uso correcto que las personas con DI hacen del preservativo cabe destacar que el $56.94 \%$ de las PDI, no se consideran capaces de utilizar de forma correcta el preservativo y un $29.52 \%$ no están seguros de si son capaces o no, frente al $24,89 \%$ de las PPG que no los considera capaces y el 21,11 que tiene dudas al respecto $\left(X^{2}=93.72 ; p=.000\right)$ (Ver figura 4).

Figura 4 Competencia del uso del preservativo entre personas con DI.

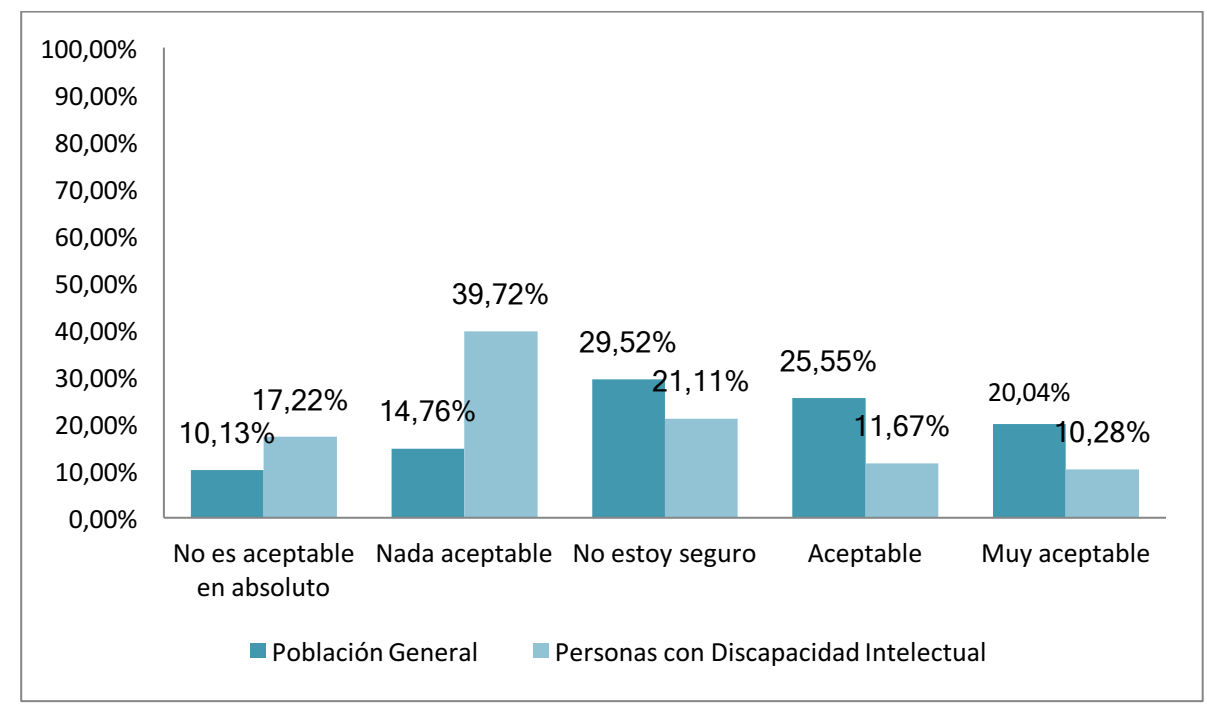

Realizando comparaciones por edad dentro de las PPG en este ítem, encontramos una distribución de puntuaciones muy parecida entre los rangos de edad más jóvenes de 20-36 años ( $M=3.34$; $D T=1.17)$ y 36-51 años $(\mathrm{M}=3.35$; DT: 1.27). Esto indica que estos grupos tienen dudas acerca de si las PDI sabrían utilizarlo correctamente. Sin embargo, el grupo de mayor edad (de 67-82 años) considera que no son capaces de usarlo correctamente y muestran una puntuación media bastante inferior $(M=1.75$; $D T=1.50)$, con diferencias estadísticamente significativas $\left(X^{2}=19.35 ; p \leq, 001\right)$.

\section{DISCUSIÓN Y CONCLUSIONES}

El objetivo de esta investigación ha sido comparar las actitudes hacia la sexualidad de las PDI que tienen ellas mismas con las que tiene la PPG. Si analizamos los diferentes tipos de prácticas sexuales vemos que las PPG valoran muy positivamente las relaciones que solo implican besos y caricias entre PDI, pero sus actitudes son más erotofóbicas cuando se trata de relaciones sexuales con penetración entre PDI. Las reticencias no parecen deberse al temor a un embarazo no deseado (Powell y Stein, 2016), ya que un gran número de personas opina que son capaces de utilizar el preservativo para la prevención de Infecciones de Transmisión Sexual y embarazos no deseados. La valoración positiva de los besos y caricias, sin embargo, puede deberse a la infantilización que en muchas ocasiones caracteriza a este colectivo (Ferrai, 2020). Por tanto, los resultados aportados en esta investigación concuerdan con otras investigaciones que concluyen que las PPG presenta actitudes hacia la sexualidad más favorables hacia aspectos generales relacionados con la libertad de expresión afectiva (besos, caricias 0 abrazos), que cuando se trata de aspectos relacionados con la maternidad/parentalidad o relaciones sexuales, donde se muestran mucho más conservadores (Hasson-Ohayon, Hertz, Vilchinsky y Kravetz, 2014; Olavarrieta et al., 2013; Franco et al., 2012). 
Nuestros resultados muestran que las PPG presenta actitudes más liberales hacia la sexualidad de las PDI que las personas del propio colectivo. En ambos grupos, la mayoría de las personas se identifica con actitudes moderadas; sin embargo, existe un porcentaje muy elevado de PDI con actitudes conservadoras y en las PPG un porcentaje más elevado con actitudes más liberales. Esto ya se observaba en estudios anteriores (MorellMengual et al., 2017), donde los autores encontraron que las PDI mostraban actitudes más negativas hacia aspectos relacionados con su sexualidad. Esto evidencia la importancia de tratar esto en los programas de concienciación social, ya que las actitudes negativas hacia la sexualidad de las personas con DI pueden provocar la restricción de la expresión de su sexualidad (Franco, Cardoso y Neto, 2012; Olavarrieta et al., 2013).

Por otro lado, centrándonos en las puntuaciones obtenidas por las PDI, ellos no se consideran del todo competentes en el uso del preservativo para prevenir Infecciones de Transmisión Sexual y embarazos, aunque muestran interés hacia las relaciones sexuales con penetración. En primer lugar debemos tener en cuenta que dentro del colectivo de personas con DI se incluye un amplio abanico de realidades desde quienes precisan pocos apoyos, y en esa medida cuentan con una mayor autonomía, hasta quienes precisan apoyos de forma continuada e intensa viendo reducidas sus posibilidades de interacción dada su mayor dependencia (APA, 2013). Así pues, en lógico pensar que muchos de ellos/as no se sientan competentes en el uso del preservativo ya es posible que no hayan tenido hasta el momento ninguna ocasión de utilizarlo. Por otra parte, como señalan algunos autores (Powell y Stein, 2016; Morell-Mengual et al., 2017; Forrai, 2020), aun estando en disposición de utilizar el preservativo, la información que se les proporciona ha sido muy limitada y/o no han podido tener oportunidades reales de poner en práctica dicha información. Así, en la investigación realizada por Moretín-Gutiérrez, AriasMartínez, Rodríguez-Mayor y Verdugo-Alonso (2012), donde comparaban diferentes parámetros de satisfacción sexual de PDI y PPG se concluye que, en general, Ios resultados son semejantes en ambos grupos y la diferencia se encuentra en el grado de permisividad del entorno que influye, de forma determinante, en la realización de diferentes prácticas sexuales y en la expresión de su sexualidad. Todo ello, provoca que sigan teniendo creencias estigmatizadas que actúan como barrera y afectan a la autoconfianza, el funcionamiento sexual y al establecimiento de nuevas relaciones (Esmail, Darry, Walter, y Knupp, 2010). En este sentido, la vivencia de la sexualidad en este colectivo está condicionada en primer lugar por los efectos directos de la discapacidad (que afectan al grado de autonomía), en segundo lugar, por la ausencia de posibilidades y condiciones adecuadas para expresar su sexualidad (de tanteo, aprendizaje y afrontamiento) y, por último, por el grado de dependencia de un servicio 0 persona (Gómez-Zapiain, 2009). La confluencia de estos factores tiene una fuerte implicación en su percepción de competencia a nivel sexual. Por estos motivos es necesario desarrollar programas de educación afectivosexual adaptados a las diferentes características/necesidades derivadas de los distintos niveles de autonomía que muestran las personas de este colectivo. Estos programas deben presentar un enfoque positivo de la sexualidad que contribuya al desarrollo óptimo de la sexualidad en las PDI (Chou, Jane, Chen y Lin, 2019; 0'Shea y Despott, 2018). Estas iniciativas deben presentar los conceptos de forma explícita y no fragmentada, inconsistente ajustándose al nivel apropiado para facilitar el correcto procesamiento de la información (Forrai, 2020). Es importante destacar que para potenciar su efectividad, estos programas deben incluir también a los profesionales que trabajan con ellos y a sus familias (Moretin, Arias, Rodríguez y Aguado, 2012), para facilitar un desarrollo sexual normativo en las PDI (Moretín-Gutiérrez et al., 2012). En un nivel de intervención más ambicioso, sería interesante desarrollar, de forma paralela a estos programas, proyectos 0 actividades destinadas a la población general que contribuyan a mejorar las actitudes hacia la sexualidad de este colectivo facilitando, de este modo, la normalización definitiva de su sexualidad.

\section{REFERENCIAS BIBLIOGRÁFICAS}

Álvarez, I., Vega, V., Spencer, H., González, F., y Arriagada, R. (2019). Adultos chilenos con discapacidad intelectual: creencias, actitudes y percepciones parentales sobre su autodeterminación. Siglo Cero, 50(2), 51-72. doi: 10.14201/scer020195025172 
American Psychiatric Association (APA). (2013). Diagnostic and statistical manual of mental disorders (5th ed.).Washington, DC: APA.

Banks, J., McCoy, S., Frawley, D., Kingston, G., Shevlin, M., y Smyth, F. (2016). Special Classes in Irish Schools Phase 2: A Qualitative Study. Dublin: Economic and Social Research Institute (ESRI) Research Series.

Bernal-Giraldo, M. J. (2016). Actitudes implícitas y explícitas en universitarios y familiares sobre Síndrome de Down (Tesis Doctoral). Pontificia Universidad Javeriana, Bogotá, Colombia.

Borawska-Charko, M., Rohleder, P. y Finlay, W. M. L. (2017). The sexual health knowledge of people with intellectual disabilities: A review. Sexuality Research and Social Policy, 14(4), 393-409.doi: 10.1007/s13178016-0267-4

Brown, M., y McCann, E. (2018). Sexuality issues and the voices of adults with intellectual disabilities: A systematic review of the literature. Research In Developmental Disabilities, 74, 124-138. doi: 10.1016/j.ridd.2018.01.009

Chou, Y. C., Jane, Z.Y., Chen, B. W., y Lin, C. J. (2019). Awareness of Sexual Rights and Empowerment: Quantitative and Qualitative Evaluation of a Sexual Health Intervention for Adults with Intellectual Disability. The Journal of Sex Research, 1-17.

Coyne, D. (2016). Practice guide for practitioners who support people with disability. Supporting Sleep Practice Guide, V1.

wirynkalo, K., Byra, S., y yta, A. (2017). Sexuality of adults with intellectual disabilities as described by support staff workers. Hrvatska revija za rehabilitacijska istraživanja, 53, 77-87.

Deffew, A. (2019). Intimate relationships and sexuality for adults with an intellectual disability: exploring the views of adult intellectual disability service providers and their staff members (Tesis Doctoral). University of Limerick, Irlanda.

Díaz-Rodríguez, I. M. (2017). Construcción de la sexualidad y análisis de su influencia en la calidad de vida y prevención de abusos sexuales en personas adultas con discapacidad intelectual (tesis doctoral). Universidad de Valencia, España.

Díaz-Rodríguez, I., Gil-Llario, M. D., Ballester-Arnal, R., Morell-Mengual, V. y Molero-Mañes, R. (2014). Conocimientos, comportamiento y actitudes sexuales en adultos con discapacidad intelectual. International Journal of Developmental and Educational Psychology, 1(3), 415-422.

Díaz-Rodríguez, I., Gil-Llario, M. D., Morell-Mengual, V., Salmerón-Sánchez, P., y Ruiz-Palomino, E. (2016). Actitudes hacia la sexualidad: ¿Difieren las personas con discapacidad intelectual de la población general?. International Journal of Developmental and Educational Psychology,

Egholm, A. (2015). Nurses' Attitudes and Beliefs towards Discussing Sexuality with Patients (Bachelor's thesis). Tampereen Ammattikorkeakoulu, Finland.

Esmail, S., Darry, K., Walter, A., y Knupp, H. (2010). Attitudes and perceptions towards disability and sexuality. Disability and rehabilitation, 32(14), 1148-1155. doi: 10.3109/09638280903419277

Forrai, J. (2020). Fogyatékkal él k szexuális egészségpolitikája, szempontjai és problémái sexual - Health policy, aspects and problems of people with disabilities. Magyar Tudomány, 181(3), 314-328. doi: 10.1556/2065.181.2020.3.4

Franco, D. G., Cardoso, J. y Neto, I. (2012). Attitudes towards affectivity and sexuality of people with intellectual disability. Sexuality and Disability, 30(3), 261-287. doi: 10.1007/s11195-012-9260-x

Gil Llario, M. D., Morell Mengual, V., Ballester Arnal, R., y Díaz Rodríguez, I. (2018). The experience of sexuality in adults with intellectual disability. Journal of Intellectual Disability Research, 62(1), 72-80. doi: 10.1111/jir.12455

Gómez-Zapiain, P. (2009). La sexualidad en las sociedades modernas: sexualidad y afectividad en personas con discapacidad intelectual - Breve guía para la reflexión. En C. Huesca, Sexualidad y discapacidad Actas Curso de Verano 2009. Zaragoza, España. 
Hasson-Ohayon, I., Hertz,I., Vilchinsky , N. y Kravetz, S. (2014). Attitudes toward the sexuality of persons with physical versus psychiatric disabilities. Rehabilitation Psychology, 59(2), 236-241.

Isler, A., Tas, F., Beytut, D. y Conk, Z. (2009). Sexuality in Adolescents with Intellectual Disabilities. Sexuality and Disability, 27(1), 27-34. doi: 10.1007/s11195-009-9107-2

Mena-Chávez, N. P. y Martínez-Herrera, J. L. (2019). Factores que dificultan el desarrollo de las personas con síndrome de down de la zona conurbada de Guadalupe y Zacatecas. IBN SINA, 10(1), 15-15.

Morell-Mengual, V., Gil-Llario, M. D., Díaz-Rodríguez, I., y Caballero-Gascón, L. (2017). Actitudes de padres, profesionales y población general hacia la sexualidad de las personas con discapacidad física e intelectual. International Journal of Developmental and Educational Psychology, 4(1), 173-183. doi: 10.17060/ijodaep.2017.n1.v4.1040

Morentin-Gutiérrez, R., Arias-Martínez, B., Rodríguez-Mayoral, J. M., y Verdugo-Alonso, M. Á. (2012). El amor en personas con discapacidad intelectual y su repercusión en el bienestar emocional.

Morentin, R., Arias, B., Rodríguez, J. M., y Aguado Díaz, A. L. (2012). Pautas para el desarrollo de programas eficaces de educación afectivo sexual en personas con discapacidad intelectual.

Olavarrieta, S., Darín, L., Suárez, P., Tur, N., Besteiro, B. y Gómez-Jarabo, G. (2013). Actitudes hacia la sexualidad, esterilización, maternidad/paternidad y habilidades de crianza de personas con discapacidad intelectual: un estudio preliminar. Siglo Cero, 44 (4), 55-69.

Organización Mundial de la Salud (2015). Sexual health, human rights and the law. Geneva: World Health Organization.

O'Shea, A., y Despott, N. (2018). LGBTIQ people with intellectual disability. Intellectual Disability Australasia, 39(3), 46.

Powell, R. M., y Stein, M. A. (2016). Persons with disabilities and their sexual, reproductive, and parenting rights: an international and comparative analysis. Frontiers of Law in China, 11(1), 53-85. doi: 10.3868/s050-005016-0005-6

Pownall, J. D., Jahoda, A. y Hastings, R. P. (2012). Sexuality and Sex Education of Adolescents with Intellectual Disability: Mothers' Attitudes, Experiences, and Support Needs. Intellectual and Developmental Disabilities, 50(2), 140-154. doi: 10.1352/1934-9556-50.2.140

Rojas-Pernia, S., Haya-Salmón, I., y Lázaro-Visa, S. (2016). Necesidades afectivo-sexuales en personas con discapacidad intelectual. Claves para construir propuestas formativas desde la experiencia subjetiva.

Rushbrooke, E., Murray, C. D., y Townsend, S. (2014). What difficulties are experienced by caregivers in relation to the sexuality of people with intellectual disabilities? A qualitative meta-synthesis. Research In Developmental Disabilities, 35(4), 871-886. doi:10.1016/j.ridd.2014.01.012

Sankhla, D., y Theodore, K. (2015). British attitudes towards sexuality in men and women with intellectual disabilities: a comparison between white westerners and south Asians. Sexuality and disability, 33(4), 429-445. doi: 10.1007/s11195-015-9423-7

Velarde-Lizama, V. (2012). Los modelos de la discapacidad: un recorrido histórico. REV-Revista Empresa y Humanismo, $15(1)$.

Wehmeyer, M. L. (1999). A functional model of self-determination: Describing development and implementing instruction. Focus on autism and other developmental disabilities, 14(1), 53-61.

Wehmeyer, M. L. (2005). Self-determination and individuals with severe disabilities: Re-examining meanings and misinterpretations. Research and Practice for Persons with Severe Disabilities, 30(3), 113-120. 